\title{
Artificial Neural Network Model for the Prediction of Thunderstorms over Kolkata
}

\author{
Litta A. J \\ Dept. of Computer Science \\ Cochin University of Science \\ and Technology, Cochin-22 \\ Kerala, India
}

\author{
Sumam Mary Idicula \\ Dept. of Computer Science \\ Cochin University of Science \\ and Technology, Cochin-22 \\ Kerala, India
}

\author{
C. Naveen Francis \\ Network System \& \\ Technologies (P) Ltd. (NeST) \\ Technopark, Trivandrum, \\ Kerala - 695 581, India
}

\begin{abstract}
Severe thunderstorms frequently occur over the eastern and north-eastern states of India during the pre-monsoon season (March-May). Forecasting thunderstorm is one of the most difficult tasks in weather prediction, due to their rather small spatial and temporal extension and the inherent non-linearity of their dynamics and physics. In this paper, experiments are conducted on artificial neural network (ANN) model to predict severe thunderstorms that occurred over Kolkata on 3 May 2009, 11 May 2009 and 15 May 2009 using thunderstorm affected parameters and validated the model results with observation. The performance of ANN model in predicting hourly surface temperature during thunderstorm days using different learning algorithms are evaluated. A statistical analysis based on mean absolute error, root mean square error, correlation coefficient and percentage of correctness is performed to compare the predicted and observed data. The results show that the ANN model with Levenberg Marquardt algorithm predicted the thunderstorm activities well in terms of sudden fall of temperature and intensity as compared to other learning algorithms.
\end{abstract}

\section{General Terms}

Neural Network Modeling, Thunderstorm Prediction, Statistical Analysis

\section{Keywords}

Artificial neural networks, learning algorithms, thunderstorm, temperature, Levenberg Marquardt.

\section{INTRODUCTION}

Thunderstorm is one of the most spectacular weather phenomena in the atmosphere. It is the towering cumulus or the cumulonimbus clouds of the convective origin and high vertical extent that are capable of producing lightning and thunder. Usually, these thunderstorms have the spatial extent of a few kilometers and life span less than an hour. However multi-cell thunderstorms developed due to organized intense convection may have a life span of several hours and may travel over a few hundreds of kilometers. Many parts over the Indian region experience thunderstorms at higher frequency during pre-monsoon months (April-May), when the atmosphere is highly unstable because of high temperatures prevailing at lower levels. Severe thunderstorms form and move generally from northwest to southeast over the eastern and northeastern states of India (i.e., Gangetic West Bengal (GWB), Jharkhand, Orissa, Bihar, Assam and parts of NorthEastern states) during the pre-monsoon season. They are locally called "Kal-baishakhi" or "Nor'westers". Strong heating of landmass during mid-day initiates convection over Chhotanagpur Plateau, which moves southeast and gets intensified by mixing with warm moist air mass from the head of Bay of Bengal (BoB). These severe thunderstorms associated with thunder, squall lines, lightning, torrential rain and hail cause extensive loss in agriculture, damage to property and also loss of life. The casualties reported due to lightning associated with thunderstorms in this region are the highest in the world. The strong wind produced by the thunderstorm is a real threat to aviation. The highest numbers of aviation hazards are reported during occurrence of these thunderstorms. In India, $72 \%$ of tornadoes are associated with Nor'westers. These severe thunderstorms have significant socio-economic impact in the eastern and northeastern parts of the country. An accurate location specific and timely prediction is required to avoid loss of lives and property due to strong winds and heavy precipitation associated with these severe local storms.

Forecasting thunderstorm is one of the most difficult tasks in weather prediction, due to their rather small spatial and temporal extension and the inherent non-linearity of their dynamics and physics. The improvement in prediction of these important weather phenomena is highly handicapped. Generally, two methods are used to forecast weather: (a) the empirical approach and (b) the dynamical approach [1]. The first is based upon the occurrence of analogues and is often referred as analogue forecasting. This is using past weather data to predict future events. The second approach is based upon equations and forward simulations of the atmosphere, and is often referred to as computer modeling (Numerical Weather Prediction). The most widely used empirical approaches for weather prediction are regression, artificial neural network, stochastic, fuzzy logic and group method of data handling. Artificial neural network (ANN) based approach can be used to model complex relationships between inputs and outputs or to find patterns in data. ANNs are trainable self-adaptive systems that can "learn" to solve complex problems from a set of examples and generalize the "acquired knowledge" to solve unforeseen problems as in stock market and environmental prediction. Previously, most weather prediction systems used a combination of empirical and dynamical techniques [2, 3, 4]. However, a little attention was paid to the use of ANNs in weather forecasting [5, 6, 7, 8, $9,10]$. The recent advances in neural network methodology for modeling nonlinear, dynamical phenomena along with the impressive successes in a wide range of applications, motivated to investigate the application of ANNs for the prediction of thunderstorms. In this paper, sensitivity experiments are carried out for studying the impact of different learning algorithms on predicting severe thunderstorm events that occurred over Kolkata $\left(22.52^{0} \mathrm{~N}\right.$, $88.37^{0} \mathrm{E}$ ) (Figure 1). The outline of the paper is as follows: Section 2 gives the description of data and methodology. Section 3 presents the description of learning algorithms. The 
results and discussion are described in section 4 and the conclusions in section 5 .

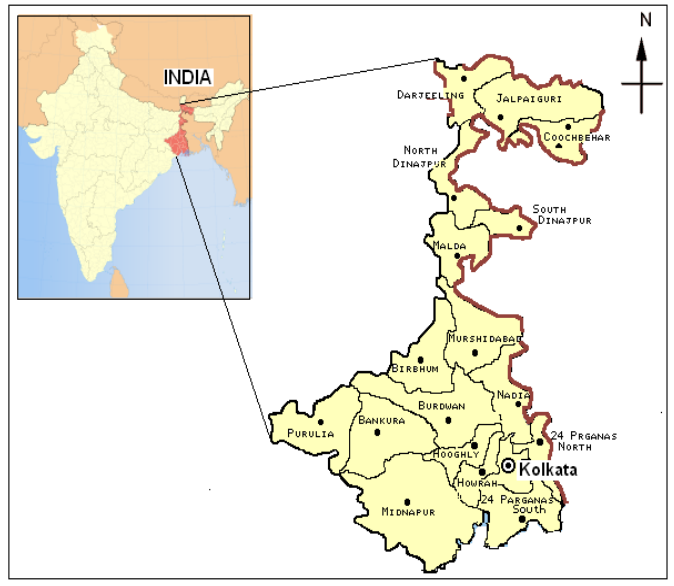

Fig 1: The geographical location of Kolkata in West Bengal

\section{DATA AND METHODOLOGY}

\subsection{ANN model configuration}

The developed ANN model is based on one of the neural network architecture named multi-layer perceptron network (MLPN). This is perhaps the most popular network architecture in use today. This is the type of network which the units each perform a biased weighted sum of their inputs and pass this activation level through a transfer function to produce their output, and the units are arranged in a layered feed forward topology. The network thus has a simple interpretation as a form of input-output model, with the weights and thresholds (biases) the free parameters of the model. Such networks can model functions of almost arbitrary complexity with the number of layers, and the number of units in each layer, determining the function complexity. Important issues in Multilayer Perceptron design include specification of the number of hidden layers and the number of units in these layers $[11,12]$. Once the number of layers and number of units in each layer have been selected, the network's weights and thresholds must be set so as to minimize the prediction error made by the network. This is the role of the learning algorithms. The best known example of a neural network learning algorithm is back propagation [13]. Modern secondorder algorithm such as conjugate gradient descent and Levenberg Marquardt are substantially faster for many problems. There are also heuristic modifications of back propagation which work well for some problem domains, such as quick propagation and Delta Bar Delta.

With this background, designed and trained the network as below: The three-layer network can represent any functional relationship between the inputs and outputs. Hence a three layer structure (one input layer, one hidden layer, one output layer) with hyperbolic tangent (tanh) transfer function for hidden layer and linear transfer function for output layer was selected (Figure 2). The hourly weather data namely mean sea level pressure, wind speed and relative humidity of 3 years (April and May 2007 to 2009) collected from the Indian meteorological department (IMD) of Kolkata, India were used as the training data. Hourly surface temperature is used as test data for ANN model. Each training and test pattern contained the time and the date of the observation. The chosen weather data were divided into two randomly selected groups, the training group, corresponding to $67 \%$ of the patterns, and the test group, corresponding to $33 \%$ of patterns. Neural networks generally provide improved performance with the normalized data. The use of original data as input to neural network may cause a convergence problem. All the weather data sets were therefore, transformed into values between -1 and 1 through dividing the difference of actual and minimum values by the difference of maximum and minimum values. At the end of each algorithm, outputs were de-normalized into the original data format for achieving the desired result. Networks were trained for a fixed number of epochs. In the present study, the outputs of ANN model with 6 learning algorithms for three thunderstorm predictions on 3 May 2009, 11 May 2009 and 15 May 2009 over Kolkata were compared. The 24h ANN predicted surface temperatures at Kolkata $\left(22.52^{0} \mathrm{~N}, 88.37^{0}\right.$ E) were used to test these models during these thunderstorm days. The capability of six different learning algorithms in predicting thunderstorms were studied and their performances were compared. The learning algorithms took for this study are Step (STP), Momentum (MOM), Conjugate Gradient (CG), Quick Propagation (QP), Levenberg Marquardt (LM) and Delta Bar Delta (DBD). Performance and reliabilities of the models were then evaluated by a number of statistical measures like root mean of squared errors (RMSE), mean absolute error (MAE) and correlation coefficients (CC).

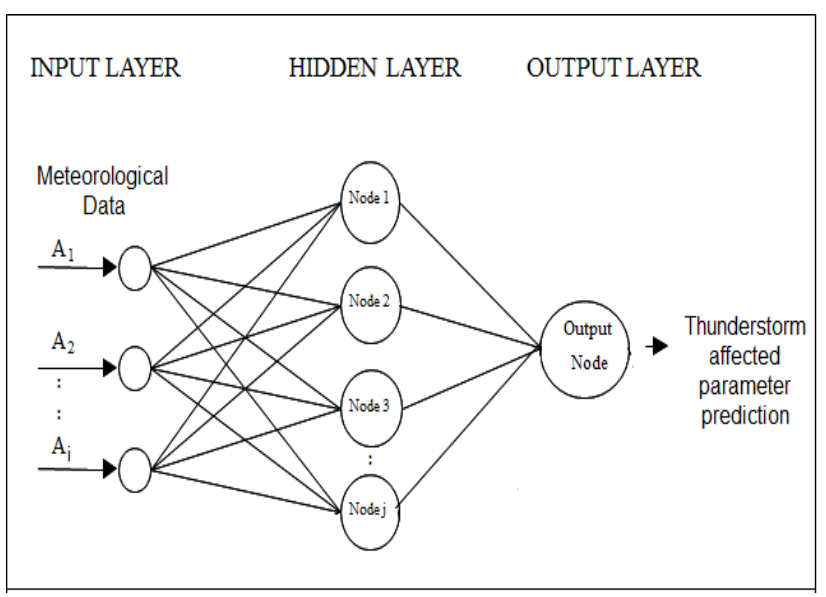

Fig 2: Architecture of multilayer perceptron network

\subsection{Statistical Analysis}

Verifying forecasts of continuous variables measures how the values of the forecasts differ from the values of the observations. The following statistical parameters were calculated for the comparisons between the forecast and observation data.

\subsubsection{Mean Absolute Error}

The mean absolute error (MAE) is the average over the verification sample of the absolute values of the differences between forecast and the corresponding observation. The MAE is a linear score which means that all the individual differences are weighted equally in the average. The MAE is a common measure of forecast error in time series analysis. It measures accuracy (the level of agreement between the forecast and the observations) for continuous variables. The MAE is given by

$$
M A E=\frac{1}{N} \sum_{i=1}^{N}\left|F_{i}-O_{i}\right|
$$

where $F_{i}$ is the forecast and $\mathrm{O}_{\mathrm{i}}$ is the observation. The difference between the forecast and the observation is the error. The lower the errors, the greater the accuracy. The range is 0 to infinity. The perfect score is 0 . 


\subsubsection{Root Mean Square Error}

The root mean square error (RMSE) is a frequently used measure of the differences between values predicted by a model and the values actually observed. It measures average error, weighted according to the square of the error. It does not indicate the direction of the deviation. The RMSE puts greater influence on large errors than smaller errors, which may be a good thing if large errors are especially undesirable, but may also conservative forecasting. The RMSE is given by

$R M S E=\sqrt{\frac{1}{N} \sum_{i-1}^{N}\left(F_{i}-O_{i}\right)^{2}}$

\subsubsection{Correlation Coefficient}

The correlation coefficient (CC) indicates the strength and direction of a linear relationship between two random variables. That means it measures the strength of the linear relationship between the forecasts and observations. The CC (r) is given by

$$
r=\frac{\sum(F-\bar{F})(O-\bar{O})}{\sqrt{\sum(F-\bar{F})^{2}} \sqrt{\sum(O-\bar{O})^{2}}}
$$

The value of $\mathrm{r}$ is such that $-1 \leq r \leq+1$. The + and - signs are used for positive linear correlations and negative linear correlations, respectively. If $F$ and $O$ have a strong positive linear correlation, $r$ is close to +1 . An $r$ value of exactly +1 indicates a perfect positive fit. A correlation greater than 0.8 is generally described as strong, whereas a correlation less than 0.5 is generally described as weak.

\section{LEARNING ALGORITHMS}

In neural network, the learning algorithms plays quite important role in the process. Throughout the process, the learning algorithm is used to adjust the weight, bias and other input parameters in such a manner that the model is able to count its best fit with the environment in a minimum amount of time. Conventional back propagation learning algorithms are often too slow for practical problems, so high performance algorithms that can converge from ten to one hundred times faster than back propagation algorithms were used. These faster algorithms fall into two main categories: The first category uses heuristic techniques developed from an analysis of the performance of the standard steepest descent algorithm. The second category uses standard numerical optimization techniques. The first category includes the gradient descent with adaptive learning rate, gradient descent with momentum, gradient descent with momentum and adaptive learning rate, and the resilient algorithm. In the standard steepest descent, the learning rate is fixed and its optimal value is always hard to find. The heuristic techniques allow the optimal learning rate to adaptively change during the learning process as the algorithm moves across the performance surface. Therefore, the performance could be improved. The second category includes CG, Quasi Newton (QN), and LM algorithm. In the CG algorithm, a search is performed along conjugate directions; therefore the convergence is faster than steepest descent directions. The QN method often converges faster than CG methods since it does not require calculation of second derivatives. For instance, it updates an approximate Hessian matrix at each iteration. The LM method combines the best features of the Gauss-Newton technique and the steepest-descent method. It also converges faster than CG methods since the Hessian Matrix is not computed but only approximated. For instance, it uses the Jacobian that requires less computation than the Hessian matrix [13].

In science and engineering problems, there are many papers in the literature that examined the effectiveness of each category of algorithms on the performance of the MLPN. For instance, authors in [14] compared the performance of LM, back propagation (BP) with momentum and $\mathrm{BP}$ with momentum and adaptive learning rate to classify the transformer oil dielectric and cooling state. They found that the BP with momentum and adaptive learning rate improves the accuracy of the BP with momentum and also gives a fast convergence to the network. The authors in [15] compared LM, CG and resilient algorithm for stream-flow forecasting and determination of lateral stress in cohesionless soils. They found that LM algorithm was faster and achieved better performance than the other algorithms in learning. The authors in [16] considered the problem of breast cancer diagnosis and compared the classification accuracy of the standard steepest descent against the classification accuracy of the gradient descent with momentum and adaptive learning, resilient BP, QN and LM algorithm. The simulations show that the neural network using the LM algorithm achieved the best classification performance. The authors in [17] demonstrated the application of ANNs in predicting the weekly spring discharge with three different learning algorithms. The learning algorithms considered by the authors were QP algorithm, batch BP algorithm, and LM algorithm. They conclude that the QP algorithm had a better performance to the application. Finally, authors in [18] compared BP, DBD, extended DBD, QP, and LM algorithms to compute the quasistatic parameters, the characteristic impedance and the effective dielectric constant, of the asymmetric coplanar waveguides (ACPWs). The results of the LM algorithm for the quasistatic parameters of the ACPWs were in very good agreement with the results available in the literature. In this study, some of these algorithms namely STP, MOM, CG, QP, LM and DBD were tried in MLPN to predict hourly surface temperature and the results are discussed.

\section{RESULTS AND DISCUSSION}

Today there are a number of parameters available that may be used to characterize pre-convective conditions and predict the beginning of convection. Johns and Doswell [19] and McNulty [20] have reviewed severe thunderstorms forecasting in detail. According to them, three of the most important factors to examine in determining occurrence of severe thunderstorm events are intense instability, a sufficiently deep humid layer in the lower and middle troposphere and an updraft to initiate convection. The formation of thunderstorms is an interaction between these conditions on different scales. The ANN model predicted results with different learning algorithms of severe thunderstorm cases are explored in the following section. Analysis of the results of these experiments is helpful to understand the impact of learning algorithms on the prediction of severe thunderstorm events and assist in the customization of model for future severe thunderstorm predictions over east and northeast Indian region.

The surface parameters play a significant role in the genesis whereas the strength of the upper air pull is required to assess the growth of the thunderstorm [21]. The greater the density differences between air masses (temperature and humidity) the greater the atmospheric instabilities that develop, and the greater the intensity of these thunderstorms [22]. Recent studies show a high positive correlation between surface temperature and lightning activity [23]. The hourly temperatures on the surface are useful tool in forecasting the 
likelihood occurrence of a thunderstorm [24]. Meteorologists warn that a sudden drop in temperature during the day indicate for thunderstorm [21].

Figure 3 shows the inter-comparison of observed and ANN model predicted diurnal variation of surface temperature $\left({ }^{0} \mathrm{C}\right)$ with different learning algorithms over Kolkata valid for 3 May 2009, 11 May 2009 and 15 May 2009. From the figures, it is clearly visible that the observed data show a sudden drop in temperature in all three thunderstorm days. The ANN model with different learning algorithms captured the sudden temperature drop during the thunderstorm hour for all the three cases. But the predicted intensity is different for different algorithms. For the first case (Figure 3a), the observed temperature showed a sudden drop of $14^{\circ} \mathrm{C}$ from $36^{\circ} \mathrm{C}$ to $22^{\circ} \mathrm{C}$ at $10 \mathrm{UTC}$. The ANN model prediction with LM showed a drop from $33^{\circ} \mathrm{C}$ to $22^{\circ} \mathrm{C}\left(11^{\circ} \mathrm{C}\right)$ at $10 \mathrm{UTC}$, whereas CG presented a drop from $34^{\circ} \mathrm{C}$ to $27^{\circ} \mathrm{C}\left(7^{\circ} \mathrm{C}\right)$ at $10 \mathrm{UTC}$. All other algorithms show a difference less than $4^{0} \mathrm{C}$ during thunderstorm hour. The DBD has a least performance than other algorithms. In the second thunderstorm case (Figure $3 \mathrm{~b}$ ), observed temperature fall is from $33^{\circ} \mathrm{C}$ to $22^{\circ} \mathrm{C}\left(11^{\circ} \mathrm{C}\right)$ at 13 UTC, whereas LM indicated a drop from $32^{\circ} \mathrm{C}$ to $21^{\circ} \mathrm{C}\left(11^{\circ} \mathrm{C}\right)$ at 13 UTC. CG showed only $6^{0} \mathrm{C}$ difference between predicted and observed values. The other algorithms presented less intensity in difference between predicted and observed values. For the third case (Figure $3 \mathrm{~b}$ ), observed temperature showed a drop from $30^{\circ} \mathrm{C}$ to $24^{\circ} \mathrm{C}\left(6^{\circ} \mathrm{C}\right)$ at $13 \mathrm{UTC}$, whereas $\mathrm{LM}$ showed a drop from $32^{\circ} \mathrm{C}$ to $26^{\circ} \mathrm{C}\left(6^{\circ} \mathrm{C}\right)$ and $\mathrm{CG}$ displayed a drop from $32^{\circ} \mathrm{C}$ to $27^{\circ} \mathrm{C}\left(5^{\circ} \mathrm{C}\right)$ at 13 UTC. The other algorithms have also captured sudden fall and intensity. From these analyses, we can see that ANN model with LM algorithm captured the sudden temperature fall with almost same drop in intensity as compared to other algorithms.

A statistical analysis based on MAE, RMSE and CC is performed for comparisons between the predicted and observed temperature with different learning algorithms valid for 3 May 2009, 11 May 2009 and 15 May 2009 (Table 1). The results indicated that, LM algorithm has less MAE and RMSE as compared to all other algorithms for these 3 thunderstorm cases. The CG algorithm has also given moderate results. All other algorithms displayed more error in all cases as compared to LM and CG algorithms. The average MAE and RMSE from these 3 cases are also less for LM algorithm than other 5 algorithms. Another verification method used for this study is correlation coefficient. From the table we can clearly see that all the algorithms are positively correlated. The LM algorithm has the highest CC in all three cases as compared to all other algorithms. The average $\mathrm{CC}$ of $\mathrm{LM}$ and $\mathrm{CG}$ algorithms are more than 0.9. The CC of other algorithms are less than 0.85 . The performance of DBD algorithm is less efficient than other algorithms.

(a) 3 May 2009

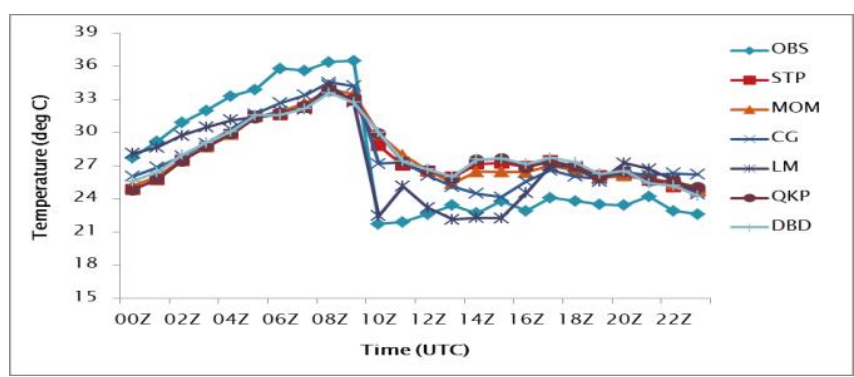

(b) 11 May 2009

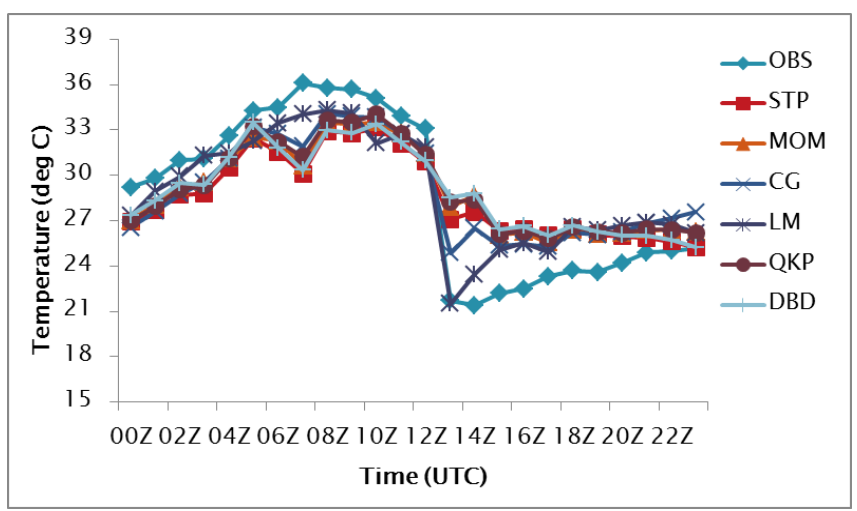

(c) 15 May 2009

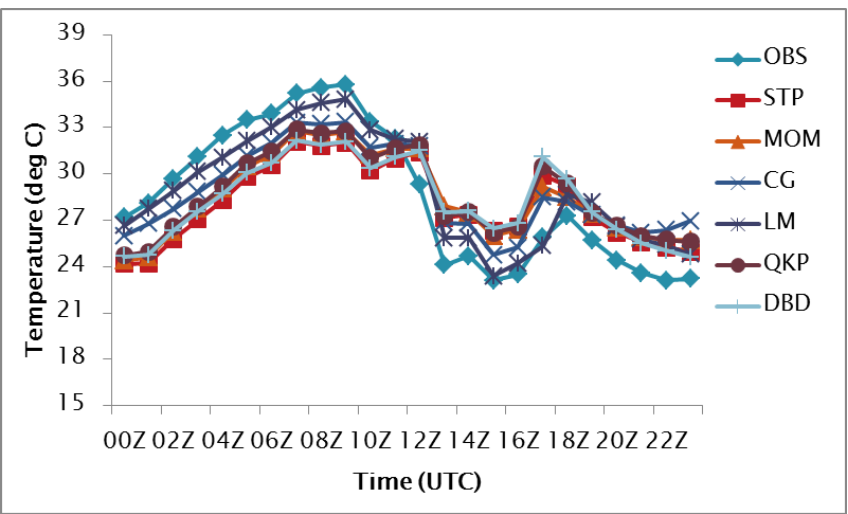

Fig 3: Comparison of ANN predicted hourly surface temperature using different learning algorithms with observation on (a) 3 May 2009 (b) 11 May 2009 (c) 15 May 2009.

Figure 4 gives the performance accuracy of learning algorithms for hourly temperature prediction. The percentage of correctness presented a percentage number of the times when the forecast is accurate to within $+/-2^{\circ} \mathrm{C}$. The result clearly indicated that overall accuracy of LM algorithm for three events is $76 \%$. CG gave a moderate accuracy of $61 \%$. Other algorithms displayed less accuracy. The time-series plots and statistical analysis of temperature revealed that LM algorithm has well predicted sudden temperature drop for the occurrence of a severe thunderstorm on all 3 thunderstorm days as in the observation.

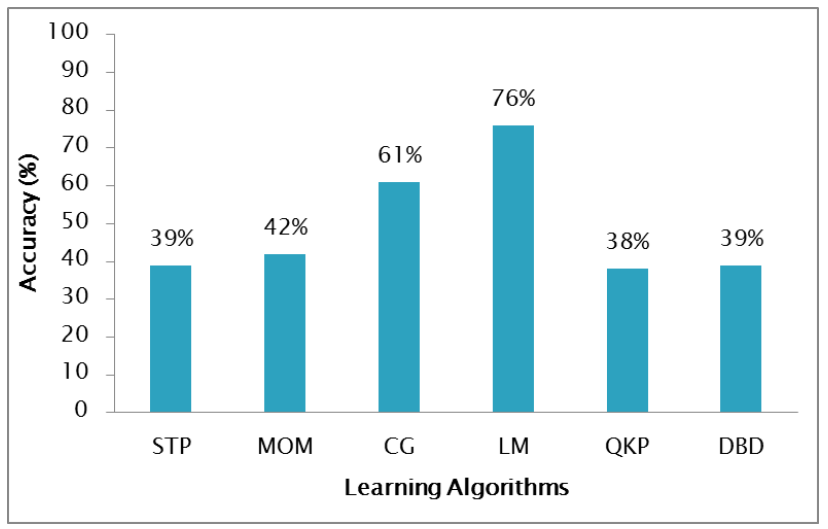

Fig 4: Performance accuracy of learning algorithms for the prediction of hourly temperature during thunderstorm days. 


\section{CONCLUSIONS}

In this paper, sensitivity experiments have been conducted with ANN model to test the impact of learning algorithms on severe thunderstorms prediction that occurred over Kolkata on 3 May 2009, 11 May 2009 and 15 May 2009 and validated the model results with observation. A statistical analysis based on mean absolute error, root mean square error, correlation coefficient and percentage of correctness is also performed for comparison among predicted and observed data with different learning algorithms. This is the first study conducted to investigate the sensitivity of learning algorithms with ANN model to predict thunderstorms over the eastern region of India. In all experiments, the model setups were identical except for the use of different learning algorithms. Hence the differences in the prediction results attributed to the sensitivity of learning algorithms. It is clearly demonstrated that LM algorithm performance is significantly better than other algorithms. After analyzing the results, we can conclude that the ANN model with LM algorithm has well predicted the hourly temperature in terms of sudden fall of temperature and intensity as compared to other learning algorithms. The results of these analyses demonstrated the capability of ANN model in prediction of severe thunderstorm events over eastern Indian region.

\section{ACKNOWLEDGMENTS}

The authors would like to express their sincere gratitude to Dr. Ajith Abraham, Machine Intelligence Research Labs, Washington 98071-2259, USA and Dr. U. C. Mohanty, Centre for Atmospheric Sciences, Indian Institute of Technology (IIT) Delhi, Hauz Khas, New Delhi - 110016 for their valuable suggestions and comments which have indeed helped us to improve our works. Thanks are due to the data providers specially India Meteorological Department (IMD).

\section{REFERENCES}

[1] E.N. Lorenz, 1969. "Three approaches to atmospheric predictability," Bulletin of the American Meteorological Society, vol. 50, pp. 345-349.

[2] Doswell, C.A., III, 1982. "The operational meteorology of convective weather, Vol. I: operational Mesoanalysis," NOAA Technical Memorandum. NSSFC-5. NOAA, National Severe Storms Forecast Center, Kansas City, MO, 135 pp.

[3] D.S. Wilks, 1995. "Statistical methods in the atmospheric sciences, an introduction," International Geophysics Series, vol. 59, Academic Press, New York, 464 pp.

[4] P.B. Mark, D.S. David, W.J.T. David, J.D. Timothy, J.C. Andrew and O.N. Alan, 2003. "Stratospheric memory and skill of extended-range weather forecasts," Science, vol. 301, pp. 636-639.

[5] D.W.A. McCann, 1992. "Neural network short-term forecast of significant thunderstorms," Weather and Forecasting, vol. 7, pp. 525-534.

[6] J. Shao, 1998. "Improving nowcasts of road surface temperature by a back propagation neural network," Weather and Forecasting, vol. 13, pp. 164-171.

[7] M.M. Elkateb, K. Solaiman and Y. Al-Turki, 1998. "A comparative study of medium-weather-dependent load forecasting using enhanced artificial/fuzzy neural network and statistical techniques," Neurocomputing, vol. 23, pp. 3-14.
[8] R.J. Kuligowski and A.P. Barros, 1998. "Localized precipitation forecasts from a numerical weather prediction model using artificial neural networks," Weather and Forecasting, vol. 13, pp. 1194-1205.

[9] I. Maqsood, I., Khan, M.R., Abraham, A., 2000. "Intelligent weather monitoring systems using connectionist models. International Journal of Neural, Parallel and Scientific Computations," vol. 10, pp.157178.

[10] S. Chaudhury and S. Chattopadhyay, 2005. "NeuroComputing based short range prediction of some meteorological parameters during the pre-monsoon season," Soft Computing, pp.349-354.

[11] M.T. Hagan. H.B. Demuth, M.H. Beale, 1996. "Neural Network Design.” PWS Publishing Company, Boston, Massachusetts.

[12] C. Bishop, 1995. "Neural Networks for Pattern Recognition," University press.

[13] S. Haykin., 1994. "Neural Networks, A Comprehensive Foundation," New York, Macmillan Publishing.

[14] Mokbnache L., Boubakeur A., 2002. "Comparison of Different Back-Propagation Algorithms used in The Diagnosis of Transformer Oil" IEEE Annual Report Conference on Electrical Insulation and Dielectric Phenomena, 244-247.

[15] Kişi Ö., Uncuoglu E., 2005. 'Comparaison of Three Back-Propagation Training Algorithms for Two Cases Studies" Indian Journal of Engineering \& Materials Sciences, 12, 434-442.

[16] Esugasini E., Mashor M. Y., Isa N. and Othman N., 2005. "Performance Comparison for MLP Networks Using Various Back Propagation Algorithms for Breast Cancer Diagnosis" Lecture Notes in Computer Science, 2005, 3682/2005, 123-130, DOI: 10.1007/11552451_17

[17] M Mohan Raju, R K Srivastava, Dinesh C S Bisht, H C Sharma and Anil Kumar, 2011. "Development of Artificial Neural- Network-Based Models for the Simulation of Spring Discharge," Advances in Artificial Intelligence Volume 2011, Article ID 686258.

[18] E.D. Übeyli and İ. Güler, 2004. "Multilayer perceptron neural networks to compute quasistatic parameters of asymmetric coplanar waveguides." Neurcomputing 62, 349-365.

[19] Johns, R. H. and Doswell, C. A., 1992. " Severe local storms forecasting. Weather and Forecasting," 7(4), 588612.

[20] McNulty, R. P., 1995. "Severe and Convective Weather: A Central Region Forecasting Challenge." Weather and Forecasting, 10, 187-202.

[21] Asnani, G. C., 2006. "Tropical Meteorology (Revised Edition)." Vol. II. Sindh Colony: Audh, Pune.

[22] Price, C., 2006. "Global thunderstorm activity, Sprites. In: M. Fullekrug et al. (eds.) Sprites," Elves and Intense Lightning Discharges, 85-99.

[23] William, E. R., 2005. "Lightning and climate: A review," Atmos. Res., 76, 272-287.

[24] Lopez, L., Garcia-Ortega and E., Sanchez, J. L., 2007. "A short-term forecast model for hail." Atmospheric Research, 83, 176-184. 
Table 1. Performance comparison of different learning algorithms for hourly temperature prediction

\begin{tabular}{|c|c|c|c|c|c|c|c|}
\hline $\begin{array}{c}\text { Statistical } \\
\text { Analysis }\end{array}$ & Dates & STP & MOM & CG & LM & QKP & DBD \\
\hline MAE & 3-May-09 & 3.36 & 3.24 & 2.69 & 2.08 & 3.48 & 3.41 \\
\hline & 11-May-09 & 2.69 & 2.57 & 2.27 & 1.72 & 2.54 & 2.62 \\
\hline & 15-May-09 & 2.93 & 2.66 & 2.08 & 1.21 & 2.69 & 2.9 \\
\hline & AVERAGE & $\mathbf{2 . 9 9}$ & $\mathbf{2 . 8 2}$ & $\mathbf{2 . 3 5}$ & $\mathbf{1 . 6 7}$ & $\mathbf{2 . 9 0}$ & $\mathbf{2 . 9 8}$ \\
\hline RMSE & 3-May-09 & 3.54 & 3.50 & 2.90 & 2.35 & 3.70 & 3.69 \\
\hline & 11-May-09 & 3.07 & 3.02 & 2.44 & 1.90 & 2.99 & 3.19 \\
\hline & 15-May-09 & 3.07 & 2.76 & 2.20 & 1.41 & 2.78 & 3.04 \\
\hline & AVERAGE & $\mathbf{3 . 2 3}$ & $\mathbf{3 . 0 9}$ & $\mathbf{2 . 5 1}$ & $\mathbf{1 . 8 9}$ & $\mathbf{3 . 1 6}$ & $\mathbf{3 . 3 1}$ \\
\hline & 3-May-09 & 0.82 & 0.82 & 0.90 & 0.93 & 0.79 & 0.80 \\
\hline & 11-May-09 & 0.89 & 0.89 & 0.94 & 0.97 & 0.89 & 0.86 \\
\hline & 15-May-09 & 0.74 & 0.80 & 0.91 & 0.96 & 0.80 & 0.74 \\
\hline & AVERAGE & $\mathbf{0 . 8 2}$ & $\mathbf{0 . 8 4}$ & $\mathbf{0 . 9 2}$ & $\mathbf{0 . 9 5}$ & $\mathbf{0 . 8 3}$ & $\mathbf{0 . 8 0}$ \\
\hline & & & & & & & \\
\hline
\end{tabular}

